\title{
Dermatoses cutanées et muqueuses des musiciens
}

\section{Kluger, Nicolas}

2017

Kluger , N 2017 , ' Dermatoses cutanées et muqueuses des musiciens ' , Annales de

Dermatologie et de Vénéréologie , vol. 144 , no. 6-7 , pp. 415-422 . https://doi.org/10.1016/j.annder.2017.03.005

http://hdl.handle.net/10138/297892

https://doi.org/10.1016/j.annder.2017.03.005

publishedVersion

Downloaded from Helda, University of Helsinki institutional repository.

This is an electronic reprint of the original article.

This reprint may differ from the original in pagination and typographic detail.

Please cite the original version. 


\title{
Dermatoses cutanées et muqueuses des musiciens ${ }^{\text {is }}$
}

\section{Skin and mucosal diseases in musicians}

\author{
N. Kluger
}

University of Helsinki and Helsinki University Central Hospital, Departments of Dermatology, Allergology and Venereology, Meilahdentie 2, PO Box 160, 00029 Hus, Finlande

Reçu le 27 décembre 2016 ; accepté le 10 mars 2017

Disponible sur Internet le 6 avril 2017

\section{MOTS CLÉS}

Dermatose

occupationnelle ;

Musique ;

Musicien ;

Traumatisme ;

Allergie ;

Dermatose de

contact
Résumé Les musiciens, qu'ils soient professionnels ou simples amateurs passionnés, sont exposés à une large variété de complications en lien avec la pratique de la musique. Les complications dermatologiques sont souvent asymptomatiques et constituent de simples stigmates, source de fierté pour le musicien. En revanche, si elles perturbent le jeu du musicien, elles doivent être traitées et prévenues. Nous avons revu ici ces lésions occupationnelles particulières qui ne sont pas toujours bien connues des dermatologues.

(c) 2017 Elsevier Masson SAS. Tous droits réservés.

\section{KEYWORDS}

Occupational dermatosis;

Music;

Musician;

Trauma;

Allergy;

Contact dermatitis
Summary Whether they are professional or amateur, musicians are exposed to a various number of complications in relation to their practice, including cutaneous ones. The latter are often a simple musical stigma and a reason for pride for the musician. If they are symptomatic and disturb the musician, they have to be treated and prevented. We reviewed here those occupational and peculiar lesions that are often not that well known to the dermatologist.

(c) 2017 Elsevier Masson SAS. All rights reserved.

\footnotetext{
is Cet article a fait l'objet d'une présentation au 24 Forum peau humaine et société, le 18 novembre 2016 à Lyon. Adresse e-mail : nicolas.kluger@hus.fi
} 


\section{Introduction}

Les musiciens, qu'ils soient professionnels dans un orchestre symphonique ou dans un groupe de rock, ou bien amateurs dans leur temps libre, sont exposés à une large variété de complications. Elles comprennent les complications neurologiques (dystonie) et musculosquelettiques (douleur, syndrome du canal carpien...), mais également auditives, oculaires, respiratoires, laryngées et cardiaques [1]. S'ajoutent également la pression lors des auditions ou avant un concert ou la vie lors d'une tournée, qui n'est pas de tout repos (alimentation pauvre, alcool, exercice réduit, stress, rythme nycthéméral modifié...) [1].

Le contact intime, appuyé, prolongé et répété de l'instrument avec la peau ou les lèvres du musicien ainsi que la posture contraignante, souvent non naturelle et donc inconfortable et la nécessité de s'entraîner, de répéter font du musicien un véritable « athlète » de la musique. Il n'est donc finalement pas étonnant que de nombreuses dermatoses occupationnelles aient été rapportées chez les musiciens. Les dermatoses des musiciens instrumentistes sont fréquentes et ne mettent pas en jeu le pronostic vital. Tant qu'elles sont asymptomatiques, elles constituent une source de fierté pour le musicien et une curiosité pour le médecin. Seules celles qui perturbent l'activité de l'artiste doivent être traitées et prévenues.

Les dermatoses peuvent être classées selon le type d'instrument (instruments à cordes, à vent ou les cuivres notamment) ou plus simplement par le type de dermatoses. Par catégorie, les dermatoses les plus fréquentes sont sans surprise les dermatoses traumatiques ou mécaniques dues à la répétition de l'application et/ou des frottements itératifs. Plus rarement, des allergies de contact à une partie de l'instrument, des infections ou l'exacerbation de dermatoses préexistantes en raison du stress psychologique généré par une activité musicale «professionnelle » [1-5].

La connaissance de ces dermatoses est importante pour permettre à l'instrumentiste de continuer à pratiquer sa passion ou son travail. Cette revue se focalisera principalement sur les dermatoses spécifiques associées à la pratique de la musique.

\section{Interroger et examiner un musicien professionnel}

La médecine des arts musicaux s'est développée sur le tard et accuse un retard si on la compare à la médecine sportive par exemple. L'observance des recommandations médicales par les musiciens est habituellement faible [6]. Pour favoriser une prise en charge optimale, lorsqu'un musicien consulte pour un problème médical a priori en rapport avec son activité musicale, il est important que le médecin soit quelque peu familier avec la terminologie musicale, une petite connaissance des instruments (Tableau 1) et surtout les implications physiques que demande chaque instrument. L'approche est naturellement plus facile si le médecin est lui-même un musicien amateur (ou plus).

Quelques points de l'anamnèse devront se focaliser sur le passé musical du patient. Ainsi, le musicien a-t-il connu des problèmes similaires antérieurement ou d'autres problèmes

\begin{tabular}{l} 
Tableau 1 Rappels des principales familles \\
d'instruments de musique. \\
\hline Instruments à cordes \\
Cordes frappées : piano, clavecin... \\
Cordes frottées : violon, alto, violoncelle, luth... \\
Cordes pincées : guitare, contrebasse... \\
Instruments à vent \\
Bois : flûte à bec, flûte traversière, clarinette, \\
hautbois, basson, saxophone... \\
Cuivres : trompette, trombone, tuba, cor... \\
Percussions \\
Batteries...
\end{tabular}

de santé en rapport avec la musique ? De quel(s) instrument(s) joue le musicien? S'il joue de plusieurs instruments, quel est son instrument de formation et son instrument actuel ? Est-ce que le musicien a procédé récemment à des ajustements ou des modifications sur son instrument (nouveau modèle ou changement complet de catégorie d'instrument) ? Suivront des questions en rapport avec la pratique, notamment des changements d'habitude avant l'apparition des symptômes : nombre de séances par jour, avec ou sans échauffement et durée des séances et des pauses. Un musicien peut passer plusieurs heures sans pause à travailler un passage particulièrement difficile ou intensifier son activité en vue d'une audition ou d'un concert.

\section{Épidémiologie}

Il existe plusieurs études épidémiologiques portant sur la prévalence et la fréquence des dermatoses associées à la pratique de la musique [2,7-11]. Cependant, elles sont difficilement comparables en raison des méthodologies variées (auto-questionnaire, examen clinique par un dermatologue), des populations étudiées (étudiants en musicologie, orchestre) et de la manière dont les données ont été classées. À titre d'exemple, certains auteurs mentionnent les dermatoses de contact sans distinguer les dermatoses irritatives et allergiques. Le Fiddler's neck, pourtant dermatose de contact par excellence, est comptabilisé à part ; parfois sa fréquence est calculée en incluant des musiciens ne jouant même pas d'instruments à cordes ! Enfin, comme le mentionnent Baccouche et al. [9], les musiciens présentent souvent plus d'une dermatose à la fois et certains préfèrent rapporter les résultats en rapport au dermatose et non aux nombres de musiciens. Il en résulte une certaine cacophonie dans les résultats (Tableau 2). Malgré tout, on peut dégager de ces études que les callosités et les dermatoses d'irritation prédominent largement.

\section{Dermatoses mécaniques ou traumatiques}

Il s'agit sans surprise des dermatoses les plus fréquemment rencontrées chez les musiciens. Elles surviennent électivement à la zone ou aux zones d'application de l'instrument sur la peau. La pression, la friction, le frottement, l'occlusion et la macération sont autant de facteurs impliqués dans la genèse de ces lésions. Chaque instrument 
Tableau 2 Caractéristiques des principales études épidémiologiques.

\begin{tabular}{|c|c|c|c|c|}
\hline & \multirow{2}{*}{$\begin{array}{l}\text { Type d'étude, cible, } \\
n=\text { nombre de } \\
\text { répondeurs }\end{array}$} & \multicolumn{2}{|c|}{ Prévalence des dermatoses } & \multirow{2}{*}{$\begin{array}{l}\text { Principales } \\
\text { dermatoses (\%) }\end{array}$} \\
\hline & & Totale, $n(\%)$ & $\begin{array}{l}\text { Associée à la } \\
\text { musique, } n(\%)\end{array}$ & \\
\hline États-Unis [2] & $\begin{array}{l}\text { Questionnaire } \\
\text { Musiciens d'un } \\
\text { orchestre } \\
n=24\end{array}$ & NP & NP & Callosités $(71)^{\mathrm{a}}$ \\
\hline Canada [7] & $\begin{array}{l}\text { Questionnaire } \\
\text { Musiciens d'un } \\
\text { orchestre } \\
n=41\end{array}$ & NP & NP (20) & NP \\
\hline Turquie [9] & $\begin{array}{l}\text { Questionnaire } \\
\text { Musiciens d'un } \\
\text { orchestre } \\
n=97\end{array}$ & $47(48)$ & NP & NP \\
\hline Tunisie [9] & $\begin{array}{l}\text { Examen clinique } \\
\text { Étudiants en musiques } \\
\text { dans les } 4 \text { instituts } \\
\text { musicaux de la Tunisie } \\
n=594\end{array}$ & $295(49,7)$ & NP & $\begin{array}{l}\text { Callosités }(63)^{\mathrm{b}} \\
\text { Anomalies de la } \\
\text { pigmentation }(14)^{\mathrm{b}} \\
\text { Dermatoses de contact } \\
\text { d'irritation }(10)^{\mathrm{b}}\end{array}$ \\
\hline Allemagne [10] & $\begin{array}{l}\text { Questionnaire } \\
19 \text { universités de } \\
\text { musiques et d'arts, } \\
2005 \\
n=412\end{array}$ & $82(20)$ & $89(22)$ & $\begin{array}{l}\text { Callosités }(58) \\
\text { Dermatoses de contact } \\
(19)^{a}\end{array}$ \\
\hline Italie [11] & $\begin{array}{l}\text { Questionnaire puis } \\
\text { examen clinique } \\
2 \text { académies de } \\
\text { musique (étudiants et } \\
\text { professeurs) } \\
n=628\end{array}$ & $199(32)$ & $129(20)$ & $\begin{array}{l}\text { Callosités des doigts } 75 \\
\text { (57\%) ou du cou } \\
31(24 \%)\end{array}$ \\
\hline
\end{tabular}

peut être responsable d'une dermatose, véritable stigmate de l'instrument. La prévalence des lésions dermatologiques augmente avec l'ancienneté de la pratique, mais surtout la durée et la rythmicité des répétitions et varie également selon les instruments $[9,10]$. Les jeunes (enfants, adolescents), les débutants ou les musiciens changeant d'instrument sont exposés à des traumatismes car les callosités ne sont pas encore développées ou en raison d'une mauvaise technique de jeu ou d'un instrument trop grand [9].

\section{Cors et callosités}

Les cors et les callosités sont les dermatoses les plus représentées [9]. Elles touchent près de deux tiers des étudiants en musique d'après une étude menée en Tunisie [9] et sont plus fréquentes chez les musiciens jouant d'un instrument à corde ou des percussions. La topographie varie selon le type d'instrument et la technique de jeu, mais touche le plus souvent les pulpes de l'index et du majeur de la main (instrument à corde), le pouce (saxophone, clarinette), l'auriculaire (trompette) ou bien les articulations métacarpophalangiennes, interphalangiennes et les éminences thénar et hypothénar (percussions). Les callosités des lèvres touchent les joueurs d'instruments à vent de la famille des bois ou des cuivres $[9,11]$. Des callosités peuvent se développer sur la partie médiane des lèvres supérieures de joueurs de hautbois, la partie inférieure des joueurs de clarinette alors que pour les joueurs de cuivres, elle sera plutôt sur les deux portions (supérieure et inférieure).

Les callosités peuvent parfois être douloureuses et alors perturber le jeu de l'instrumentiste. Des bulles de friction sont rares, mais possibles initialement (Fig. 1) $[9,10]$. Elles ne doivent être traitées que quand elles sont excessives ou symptomatiques, notamment en cas de rhagades [9].

\section{Hyperpigmentation et hypopigmentation}

Des lésions hyperpigmentées, sous la forme de plaques mal délimitées irrégulières et floues peuvent être notées au site 


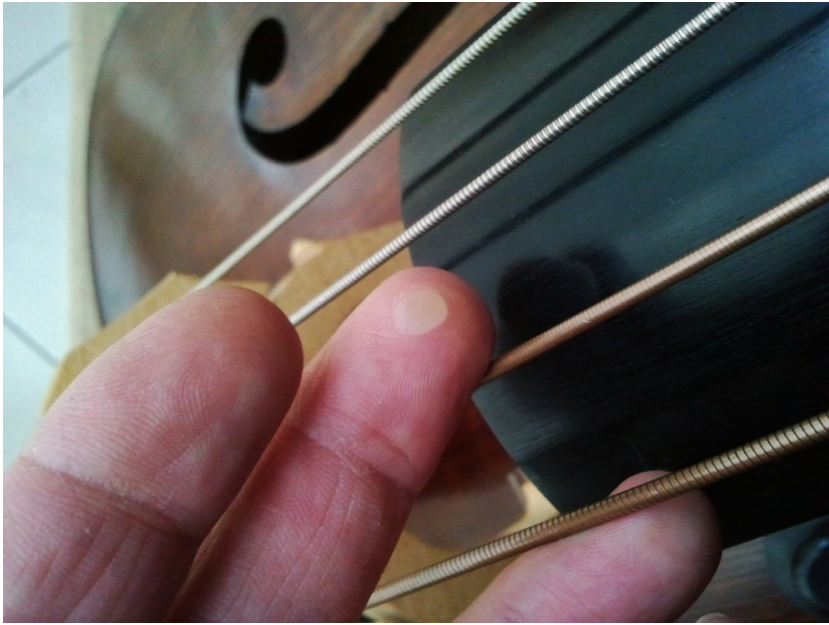

Figure 1. Phlyctène du majeur peu sensible se résorbant en 2-3 jours et survenant entre 3 à 5 fois par an lors de concerts chez un guitariste de jazz (Collection Dr Jullin).

d'application de l'instrument (cou ou région sternoclaviculaire gauches chez le violoniste) ou sur les faces internes de bras (frottement du bras sur l'instrument) [2,9]. Une coloration noire des lèvres était rapportée chez une flûtiste qui utilisait également un traitement local contre une acné périorale. La coloration noire serait due à une réaction chimique entre la solution anti-acné contenant divers oxydes et la pièce en argent de la flûte [12]. Des lésions hypopigmentées des lèvres ont été décrites lors de la pratique d'instruments à vent (clarinette, saxophone, trompette) [9] .

\section{Dystrophies unguéales}

Une fragilité des ongles de la main gauche (tenant le chevalet) avec friabilité et onychoschizie peut se rencontrer chez les violonistes notamment en cas de pratique intense [13]. Des dépressions linéaires transverses du pouce droit ont été décrites chez un joueur de guitare par frottement du pouce sur les cordes [14]. Une acro-ostéolyse a été rapportée chez un guitariste [15]. Une paronychie peut se développer chez les joueurs de guitare et de contrebasse pratiquant la technique du « pizzicato » (pincement des cordes avec les doigts de la main droite au lieu d'utiliser l'archet) («pizzicato paronychia $»$ [9].

\section{Lésions spécifiques}

Un certain nombre d'entités ont fleuri à partir des années 1970 attribuant un symptôme, un signe clinique ou une complication à un instrumentiste particulier. Il s'agit, dans la très grande majorité des cas, des pathologies mécaniques ou irritatives, parfois compressive en cas de thrombose vasculaire.

\section{Le Fiddler's neck}

Le cou du violoniste (Fiddler's neck) est probablement la lésion dermatologique la plus connue et la plus décrite

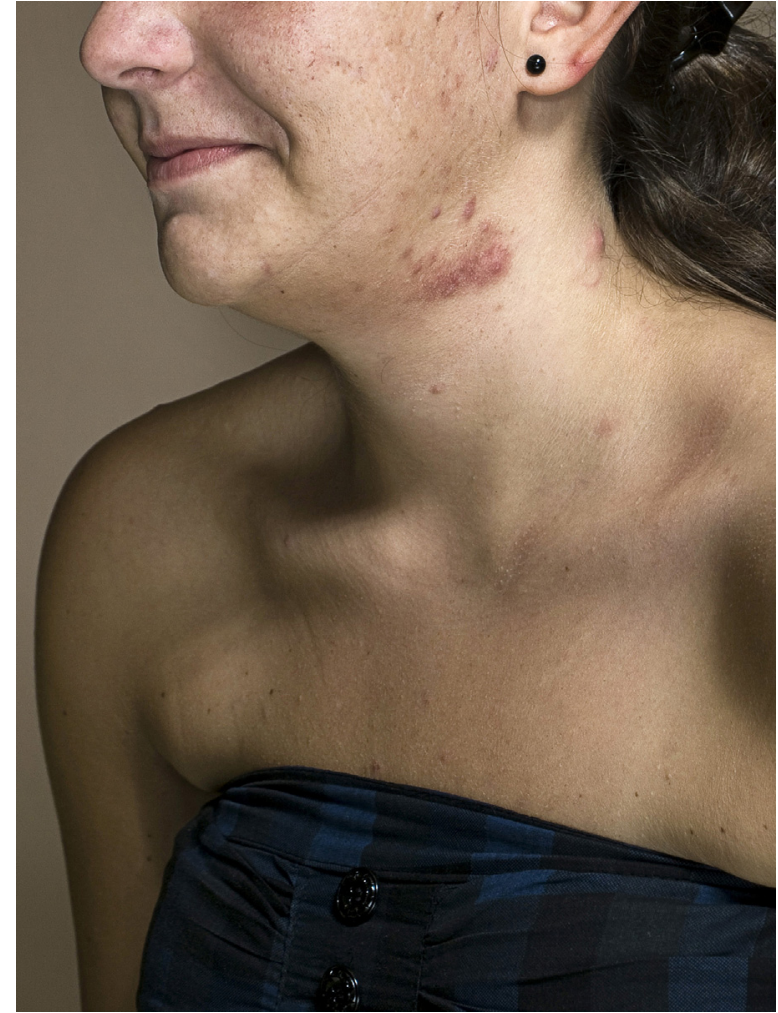

Figure 2. Fiddler's neck chez une jeune violoniste. Notez la lésion principale du cou sur fond d'acné, mais également les lésions hyperpigmentées à la partie inférieure du cou et au-dessus de la clavicule (Collection Adrie Mouthaan, photographe, Amsterdam). Photographie publiée avec l'aimable autorisation du photographe Adrie Mouthaan ${ }^{\odot} 2017$.

aussi. Peachey et Matthews en ont fait une description très précise dans un article du British Journal of Dermatology en 1978 chez 22 musiciens [16]. Elle touche plus souvent les altistes que les violonistes en raison de la taille de l'instrument. Le Fiddler's neck se présente sous la forme d'une zone de lichénification du cou, bien délimitée, unilatérale, sous l'angle de la mandibule gauche - le jeu du violon étant indépendant de la latéralité du violoniste - à l'endroit même où le violoniste repose son cou sur la mentonnière du chevalet. La zone peut se présenter également sous la forme d'une macule ou d'une plaque hyperpigmentée, d'un érythème, de papules inflammatoires, de pustules ou de lésions kystiques (Fig. 2) [16]. Une alopécie localisée de la barbe a été décrite également [8]. Un œdème localisé du cou peut être noté, notamment après plusieurs heures de pratique, ou une adénopathie localisée récidivante [2].

L'histologie cutanée peut montrer une hyperkératose, une acanthose, un infiltrat de cellules histiocytaires avec des granulomes à corps étrangers et des kystes folliculaires. Le mécanisme étiopathogénique est multifactoriel, lié à l'expérience du violoniste, le poids de l'instrument (plus fréquent avec un alto qu'un violon) et une mentonnière inadaptée. La pression sur le violon, la friction accrue, l'occlusion et la macération due à la transpiration contribuent à la genèse de ces lésions qui rappellent une acné mécanique localisée. Un manque d'hygiène est un facteur additionnel. Le traitement comprend l'arrêt de la pratique du violon, une 
corticothérapie locale et l'application de silicone entre la peau et l'instrument. Si les lésions sont acnéiformes, le traitement est celui d'une acné (antibiotiques type cyclines). L'excision chirurgicale de la lésion est réalisée en dernière ligne [2]. Le dos des violons et des altos appuie également sur la région sternoclaviculaire et peut donc être responsable de lésions de frottement à cet endroit. Comme pour le Fiddler's neck, la prévention passe par l'utilisation de coussin pour prévenir la pression de l'appui.

\section{Les callosités du violoniste (Garrod's pads ou violonist's pads)}

Ces callosités se développent en regard des articulations interphalangiennes proximales et distales de la main gauche chez le violoniste $[8,17]$. Elles seraient la conséquence de la mise sous tension des tendons extenseurs (contraction/relaxation) lors du jeu en extrême flexion.

\section{La poitrine et les genoux des violoncellistes}

Les violoncellistes développent eux des lésions pré-sternales et aux faces internes des genoux d'où les dénominations anglo-saxonnes respectives de cello's ou cellist's chest [18] et cello ou cellist's knee. Il s'agit de lésions hyperpigmentées par frottement chronique de l'instrument et des équivalents du Fiddler's neck. En revanche, le scrotum du violoncelliste (cello scrotum) mentionné dans de nombreux articles de revue, n'est qu'une pure invention, un canular médical de ceux qui l'ont rapporté en 1974 [19]. Le pot-auxroses a été révélé par les auteurs eux-mêmes 34 ans après [20].

\section{Le mamelon du guitariste (guitar nipple)}

En 1974, Curtis rapportait le cas de 3 filles âgées de 8 et 10 ans qui présentaient des signes de mastite kystique traumatique unilatérale à la base de l'aréole mammaire en contact avec le bord du corps d'une guitare acoustique. La mastite régressait à l'arrêt de la pratique de la guitare [21]. L'utilisation d'une guitare trop grande pour l'âge pourrait expliquer les symptômes ; cependant, à notre connaissance, aucun autre cas n'a été rapporté depuis.

\section{L'aine du guitariste (guitarist's groin)}

En réponse au cas précédent [21], Semple et Gillingham ont rapporté le cas d'un jeune homme de 24 ans qui a développé une thrombose veineuse de la jambe gauche attribuée à la pratique quotidienne de la guitare $(4-6 \mathrm{~h} /$ jour, la jambe gauche en complète flexion, et la caisse de résonance de la guitare comprimant la cuisse) [22].

\section{Les doigts du harpiste (harpist's fingers)}

Le harpiste est probablement le musicien le plus exposé à des traumatismes lors de son jeu : cals, paronychies, hémorragies sous-unguéales, onycholyses forment les doigts de la harpiste [2]. Des bulles peuvent survenir initialement chez les débutants [23].

\section{La chéilite du clarinettiste (clarinettist's cheilitis)}

Quelques rares cas de chéilites ont été décrits chez les clarinettistes, allergiques ou non (Fig. 3). Cependant, les explorations allergologiques comprenant la batterie standard, les plastiques, les bois et les anches des instruments utilisés peuvent être négatives [24]. Les mécanismes irritatifs combinent la pression locale, la friction, l'occlusion et le cisaillement [2].

\section{Le menton du flûtiste (flautist's chin)}

Il s'agit d'une forme de dermatite périorale localisée sur le menton, dont la chronologie de survenue suit les périodes de jeu de flûte. Elle serait liée à la friction, le contact avec l'instrument et la salive [25]. Un traitement par cyclines est efficace. Pour certains, il pourrait s'agir parfois d'une dermatose allergique. Les hommes flûtistes peuvent se faire pousser une barbiche pour contourner le problème [2].

\section{Le purpura du glissando au piano}

Nous avons observé le cas d'une patiente musicienne qui présentait un purpura unguéal sur les 2, 3 et $4^{\mathrm{e}}$ doigts de la main droite alors que celle-ci avait répété intensivement des passages comportant des glissandos, c'est-à-dire un glissement continu d'une note à une autre en faisant traîner la main rapidement sur les touches [26]. Les lésions se résolvaient d'elles-mêmes lors du suivi de la patiente. Les manifestations cutanées au piano sont exceptionnelles et on les retrouve mentionnées dans des vieux traités médicaux [27]. Des lésions eczématiformes périarticulaires et des plis de l'index dus à la friction et à la sudation, de même que l'atrophie et l'assouplissement de l'annulaire et du pouce droit par pression excessive sur les touches. Onychodystrophie [11] et paronychie [3] peuvent également toucher les pianistes.

\section{Le syndrome de Satchmo}

«Satchmo » est un des surnoms de Louis Armstrong. Le syndrome éponyme est à mettre au crédit d'un chirurgien plasticien barcelonais [28] en rapport aux symptômes dus à un amincissement, une déchirure ou une rupture du muscle orbiculaire des lèvres [29]. L'atteinte de ce muscle constitue un handicap majeur pour les trompettistes, mais également pour tous les cuivres (tuba, trombone, cor d'harmonie) [29]. Louis Armstrong aurait souffert lui-même de cette complication l'obligeant à une pause dans sa carrière [28]. Les 

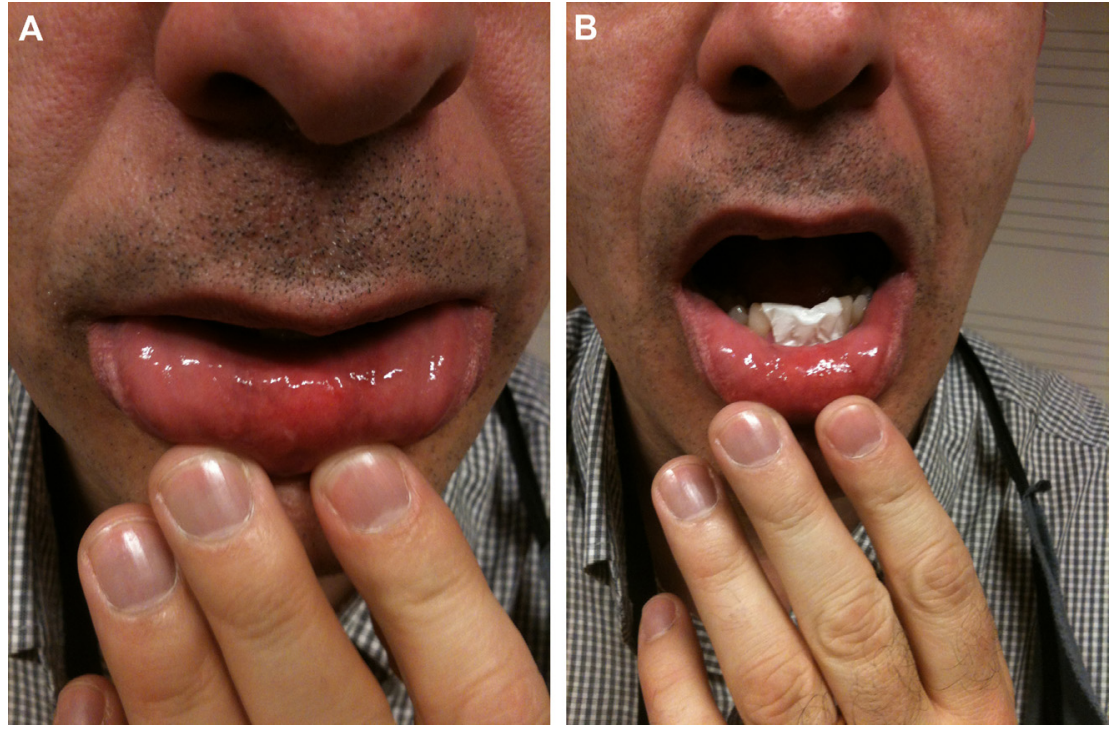

Figure 3. a : chéilite irritative sur le versant muqueux de la lèvre inférieure chez un clarinettiste ; $b$ : prévention par application d'un papier type papier à cigarette ou papier plastifié sur les incisives inférieures (Collection Dr Guillot).

musiciens se plaignent de douleur ou de faiblesse ainsi qu'une détérioration tonale, avec difficultés à jouer des notes hautes. À l'examen, il est incapable de faire une moue avec ses lèvres ou de garder de l'air sous pression dans sa bouche [28]. Le traitement est soit le repos au très long cours ou bien et surtout, une réparation chirurgicale directe $[28,29]$. Notons une curiosité mentionnée rapidement par Peachey et Matthews, le relâchement des joues chez les joueurs de tuba [16].

\section{Le syndrome de l'archet}

Le syndrome de Paget-Schroetter est une thrombose veineuse profonde primitive du membre supérieur, axillaire ou sous-clavière, liée à un mouvement répétitif. Ce syndrome n'est pas propre aux musiciens [30] mais a été rapporté chez les violonistes [30], les altistes [31] et les violoncellistes [32]. Il se présente sous la forme d'un œdème unilatéral et une sensation de lourdeur du bras droit avec érythrocyanose de déclivité et circulation collatérale dans la région de l'épaule, de la région pectorale et mammaire. La douleur ou l'inconfort du membre est soulagée par la surélévation. Le diagnostic peut être difficile et l'échographie Doppler prise à défaut initialement [30]. Dans le cas des violonistes, une intensification du jeu et des modifications dans la manière de jouer sont des facteurs déclencheurs [30,31]. D'autres facteurs peuvent s'intriquer (contraception, activité physique ou activité professionnelle avec le bras levé de façon répétitive). Le traitement repose sur l'anticoagulation. Le « syndrome de l'archet » a été récemment proposé par Sanson et al. pour décrire cette complication chez les musiciens [30].

\section{Dermatoses allergiques et irritatives}

Les dermatoses de contact allergiques et irritatives sont possibles chez les musiciens instrumentistes. Bien que rares
[11], les dermatoses allergiques doivent être évoquées devant un tableau eczématiforme aigu ou chronique aux zones de contact avec l'instrument (Fig. 4) [5,9]. La macération et l'irritation chronique peuvent bien sûr promouvoir le développement d'une allergie de contact [11]. Les allergènes sont les métaux ou les bois exotiques composant une partie ou tout un instrument, la colophane de la mèche des archets, etc. [5]. Des dermatoses irritatives peuvent se rencontrer notamment chez les cuivres (solvants, nettoyants, produits de polissage...). Les principaux allergènes sont résumés dans le Tableau 3 [4,5,33]. Parfois les diagnostics de dermatoses traumatiques et allergiques peuvent se combiner [34,35]. Enfin, il faut aussi rechercher tout autre facteur intercurrent responsable de cette dermatose. Ainsi, une flûtiste présentait une chéilite de contact au géraniol due à un stick à lèvres photoprotecteur appliqué en raison d'un herpès récidivant [36].

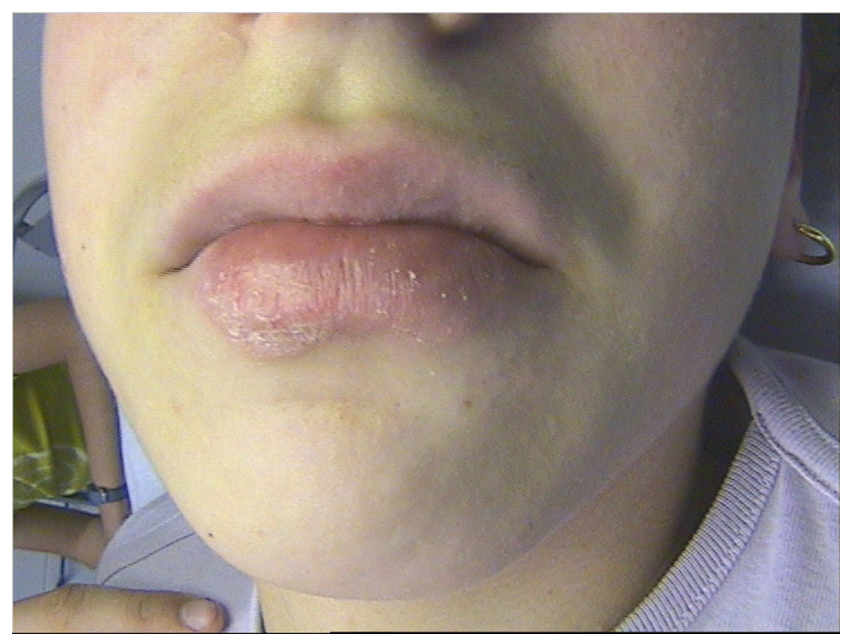

Figure 4. Chéilite allergique à une anche de saxophone (Collection Dr Trouche). 


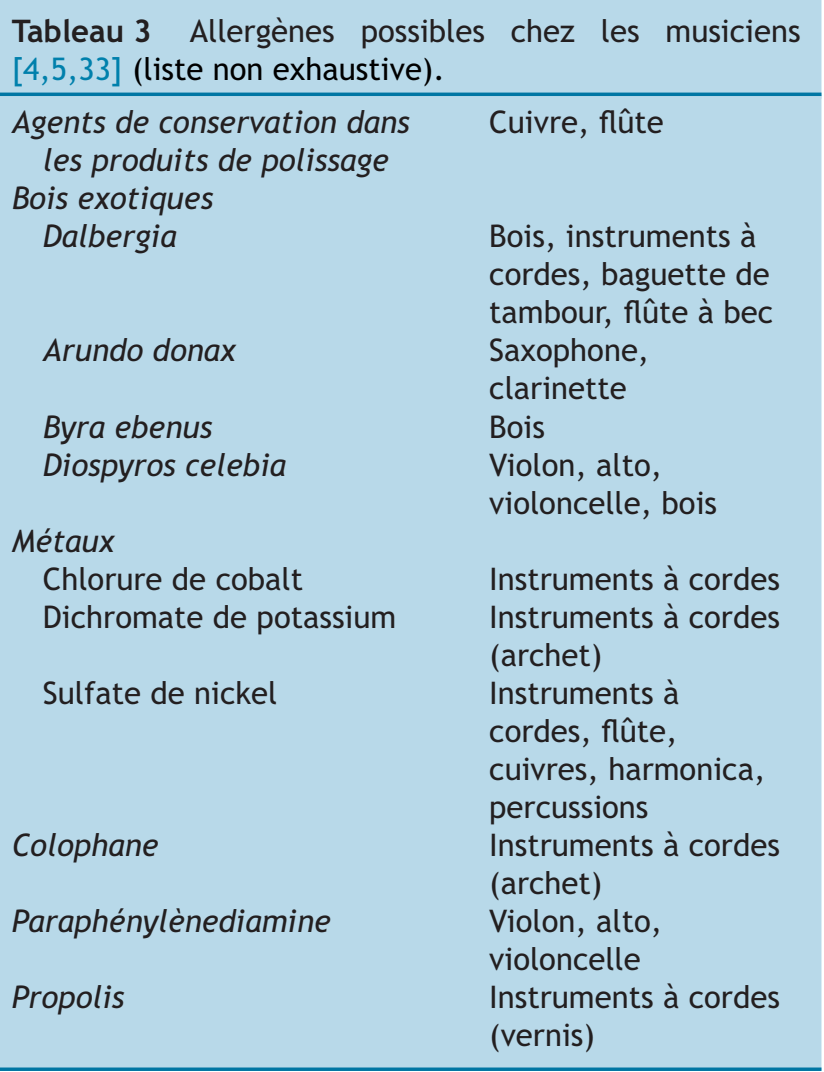

\section{Infections cutanées}

Les infections cutanées sont exceptionnelles à notre connaissance. Elles surviennent sur une peau déjà traumatisée ou par échange d'instrument. Les embouchures des instruments à vent sont cependant personnelles et ne se prêtent pas entre musiciens. Les herpès labiaux seraient plus fréquents chez les joueurs de cuivre d'après une vieille étude scandinave [37].

\section{Autres complications dermatologiques}

Des dermatoses préexistantes peuvent être exacerbées ou même révélées par la pratique de la musique. Ainsi, la dermatite atopique ou le psoriasis ne sont pas rares chez les musiciens [10]. Une exacerbation de la dermatose était rapportée chez $13 \%$ des patients dans l'étude de Gambichler et al. [10]. L'exacerbation est à mettre en rapport avec le stress psychologique généré par une activité musicale professionnelle, notamment chez les solistes. Elles n'empêchent, cependant, que rarement la pratique de la musique. Vingt pour cent des musiciens rapportent qu'elles n'affectent que de façon légère à modérée le musicien [10]. Relatons le cas d'un angio-oedème vibratoire chez un saxophoniste qui se révélait par un œdème prurigineux de la lèvre inférieure survenant quelques minutes après avoir commencé à jouer. Le diagnostic était confirmé par un test avec un vortex sur l'avant-bras et les symptômes améliorés par la prise de $5 \mathrm{mg}$ de prednisolone une heure avant de jouer [38]. L'hyperhidrose excessive peut induire une corrosion des instruments alors qu'une xérostomie va handicaper

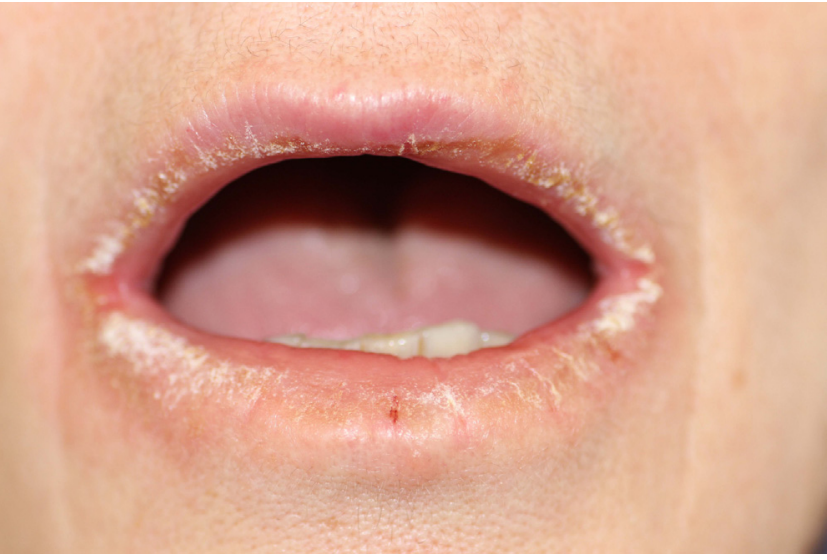

Figure 5. Chéilite mycosique, traitée par dermocorticoïdes dans l'hypothèse d'une chéilite allergique, ayant empêché une trompettiste de jouer pendant 15 jours (Collection Dr Guillot).

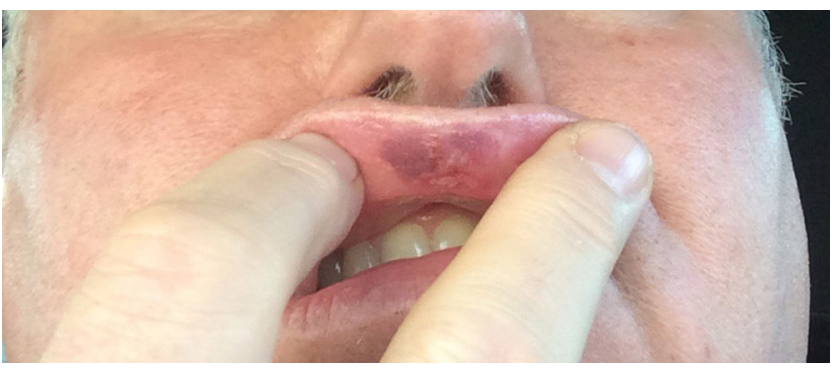

Figure 6. Hématome post-traumatique de la lèvre supérieure (indépendante de l'activité musicale) chez un trompettiste professionnelle à 48 heures d'un concert pouvant compromettre la représentation (Collection Dr Guillot).

les joueurs d'instrument à vent [2]. Ces deux pathologies ne sont pas induites par les instruments mais en relation encore une fois avec le stress et l'anxiété lors des concerts ou des auditions [2]. Enfin, rappelons que n'importe quelle dermatose ou tout traumatisme peut interférer avec le jeu des musiciens (Fig. 5). Des lésions d'allure totalement anodines peuvent avoir des conséquences dramatiques et ne doivent pas être minimisées par le médecin (Fig. 6).

\section{Conclusion}

Les dermatoses des musiciens sont multifactorielles, combinant des facteurs mécaniques, chimiques et irritatifs voire allergiques. Le dermatologue est en bonne place pour poser le diagnostic et proposer un traitement adapté au musicien. En parallèle, ce dernier doit revoir et améliorer les aspects de sa préparation et de sa technique musicale pour prévenir ces complications.

\section{Remerciements}

L'auteur souhaite remercier les $\mathrm{Dr}$ Fabienne Trouche (Rodez), Rolland Jullin (Gradignan) pour avoir partagé leurs clichés, le Dr Patrick Guillot (Bordeaux) pour ses clichés ainsi que ses conseils sur l'article, ainsi que le photographe néerlandais Adrie Mouthaan 
(http://adriemouthaan.nl/fiddlers-neck) et bien évidemment les musiciens ayant accepté de poser avec leurs stigmates musicaux.

\section{Déclaration de liens d'intérêts}

L’auteur déclare ne pas avoir de liens d'intérêts.

\section{Références}

[1] Bache S, Edenborough F. A symphony of maladies. BMJ 2008;337:a2646.

[2] Rimmer S, Spielvogel RL. Dermatologic problems of musicians. J Am Acad Dermatol 1990;22:657-63.

[3] Gambichler T, Boms S, Freitag M. Contact dermatitis and other skin conditions in instrumental musicians. BMC Dermatol 2004;4:3.

[4] Vine K, DeLeo V. Dermatologic manifestations of musicians: a case report and review of skin conditions in musicians. Cutis 2011;87:117-21.

[5] Crépy MN. Skin diseases in musicians. Eur J Dermatol 2015;25:375-83.

[6] Dommerholt J. Performing arts medicine - instrumentalist musicians part I - general considerations. J Body Mov Ther 2009;13:311-9.

[7] Nethercott JR, Holness DL. Dermatologic problems of musicians. J Am Acad Dermatol 1991;25:870.

[8] Onder M, Aksakal AB, Oztaș MO, Gürer MA. Skin problems of musicians. Int J Dermatol 1999;38:192-5.

[9] Baccouche D, Mokni M, Ben Abdelaziz A, Ben Osman-Dhahri A. Dermatoses des musiciens : étude prospective chez des étudiants en musique. Ann Dermatol Venereol 2007;134:445-9.

[10] Gambichler T, Uzun A, Boms S, Altmeyer P, Altenmüller E. Skin conditions in instrumental musicians: a self-reported survey. Contact Dermatitis 2008;58:217-22.

[11] Patruno C, Napolitano M, La Bella S, Ayala F, Balato N, Cantelli $\mathrm{M}$, et al. Instrument-related skin disorders in musicians. Dermatitis 2016;27:26-9.

[12] Koransky JS. Dermatologic problems of musicians: iatrogenic black dermographism. J Am Acad Dermatol 1994;31:519.

[13] Piraccini BM, Antonucci A, Iorizzo M, Pazzaglia M, Tosti A. Occupational nail fragility in a professional violist. Contact Dermatitis 2004;51:35-6.

[14] Wu JJ. Habit tic deformity secondary to guitar playing. Dermatol Online J 2009;15:16.

[15] Baran R, Tosti A. Occupational acroosteolysis in a guitar player. Acta Derm Venereol 1993;73:64-5.
[16] Peachey RD, Matthews CN. 'Fiddler's neck'. Br J Dermatol 1978;98:669-74.

[17] Bird HA. Development of Garrod's pads in the fingers of a professional violinist. Ann Rheum Dis 1987;46:169-70.

[18] Mandell HN. Cellist's chest. N Engl J Med 1962;266:348.

[19] Murphy JM. Letter: cello scrotum. Br Med J 1974;2:335.

[20] Murphy E, Murphy JM. Cello scrotum confession. Murphy's lore. BMJ 2009;338:b288.

[21] Curtis P. Letter: guitar nipple. Br Med J 1974;2:226.

[22] Semple R, Gillingham J. Letter: musical bumps. Br Med J 1974;2:504.

[23] Cohen PR. Harpist's finger: case report of a trauma-induced blister in a beginner harpist and review of string instrumentassociated skin problems in musicians. Cutis 2008;82:329-34.

[24] Hindson TC. Clarinettist's cheilitis. Br Med J 1978;2:1295.

[25] Dahl MG. Flautist's chin: a companion to fiddler's neck. Br Med J 1978;2:1023.

[26] Kluger N. Piano glissando purpura: another cutaneous curiosity in musicians. J Eur Acad Dermatol Venereol 2016;30:683.

[27] Schwartz L, Tulipan L, Birmingham DJ. Analysis of skin hazards in various occupations. In: Schwartz L, Tulipan L, Birmingham DJ, editors. Occupational diseases of the skin. 3rd edn. Philadelphia, USA: Lea and Febiger; 1957. p. 764-930.

[28] Planas J. Rupture of the orbicularis oris in trumpet players (Satchmo's syndrome). Plast Reconstr Surg 1982;69:690-3.

[29] Papsin BC, Maaske LA, McGrail JS. Orbicularis oris muscle injury in brass players. Laryngoscope 1996;106:757-60.

[30] Sanson H, Gautier V, Stansal A, Sfeir D, Franceschi C, Priollet $\mathrm{P}$. Thrombose veineuse profonde du membre supérieur chez une violoniste : « le syndrome de l'archet ». J Mal Vasc 2016;41:396-402.

[31] Reina NJ, Honet JC, Brown OW, Beitman M, Chodoroff G. PagetSchroëtter syndrome in a viola player. Med Probl Perform Art 1988;3:24-5.

[32] Yan BP, Kiernan TJ, Gupta V, Schainfeld RM, Garasic JM. Images in cardiovascular medicine. Over rehearsed: a cellist with Paget-Schroëtter. Circulation 2008;118:e160-1.

[33] Feldmeyer L. Dermatoses des musiciens. Nouv Dermatol 2012;31:20-2.

[34] Pincelli C, Motolese A, Pincelli L. Fiddler's neck and nickel dermatitis. Contact Dermatitis 1985;13:37.

[35] Caero JE, Cohen PR. Fiddler's neck: chin rest-associated irritant contact dermatitis and allergic contact dermatitis in a violin player. Dermatol Online J 2012;18:10.

[36] Chang YC, Maibach HI. Pseudo flautist's lip: allergic contact cheilitis from geraniol. Contact Dermatitis 1997;37:39.

[37] Barkvoll P, Attramadal A. Recurrent herpes labialis in a military brass band. Scand J Dent Res 1987;95:256-8.

[38] Patruno C, Ayala F, Cimmino G, Mordente I, Balato N. Vibratory angioedema in a saxophonist. Dermatitis 2009;20:346-7. 